consecutive patients who underwent holmium laser enucleation of the prostate to treat $\mathrm{BPH}$ at a single center.

Participants (aged 40-90 years) were divided into three groups according to their prostate size on preoperative transrectal ultrasonography (<75 g, 75-125 g and $>125 \mathrm{~g}$ ). Outcomes in all three groups were equally good, which indicated that holmium laser enucleation of the prostate is as effective for large prostates as it is for small ones. Across the whole cohort, American Urological Association symptom scores improved by 13.9, maximum flow rate increased by $9.1 \mathrm{ml}$ (a high value, given the fact that $\mathbf{2 0 0}$ men had acute urinary retention and were catheter-dependent at enrollment), and PSA levels declined by $86 \%$ compared with pretreatment values. Strictures and transient urinary incontinence were the most common complications, but were unrelated to prostate size. Most patients could have their catheter removed within $24 \mathrm{~h}$ of treatment.

The authors highlight the versatility, hemostatic control, and lack of transurethral resection syndrome achieved by holmium laser enucleation of the prostate. However, transurethral resection of the prostate has also moved on: the search for an optimum therapy for $\mathrm{BPH}$ will continue.

Original article Humphreys MR et al. (2008) Holmium laser enucleation of the prostate-outcomes independent of prostate size? J Urol 180: 2431-2435

\section{Simulator-acquired laparoscopic skills are transferable between procedures}

A study from the University of Texas has found that laparoscopic simulator training can improve the ability of students to perform urological procedures, even when the training simulation is for a completely unrelated procedure. The results of the study indicate that the skills learned on laparoscopic simulators are transferable between procedures.

Lucas et al. enrolled 32 first-year and secondyear medical students, none of whom had previous laparoscopic experience. All 32 students performed a laparoscopic cholecystectomy on a LAP Mentor ${ }^{\mathrm{TM}}$ simulator (Simbionix USA Corp., Cleveland, OH). Performance was assessed using the Objective Structural Assessment of Technical Skills (OSATS). The students were then randomly assigned to a program of six unsupervised $30 \mathrm{~min}$ training sessions, or no training. The students who underwent training were encouraged to practice lens manipulation, hand-eye coordination exercises, grasping, clipping, two-handed maneuvers, cutting, fulguration, and object translocation. At the end of the training period, all 32 students, after watching a demonstration of the procedure, performed a porcine laparoscopic nephrectomy. The OSATS scores for the initial cholecystectomy simulation were similar in both groups; however, for the nephrectomy, the students who received simulator training significantly outperformed those who did not (mean OSATS score $21.0 \pm 6.8$ vs $15.7 \pm 6.6$ ).

The authors conclude that general laparoscopy skills developed on a simulator might be applicable to unrelated procedures. As the present study involved novices, the potential value of such training in more-experienced operators should be investigated.

Original article Lucas SM et al. (2008) Training on a virtual reality laparoscopic simulator improves performance of an unfamiliar live laparoscopic procedure. J Urol 180: 2588-2591

\section{Irrigation with isoproterenol during ureterorenoscopy reduces pelvic pressure}

Irrigation during ureterorenoscopy increases renal pelvic pressure, and can potentially injure the kidney. Irrigation with isoproterenol (isoprenaline), a $\beta$-adrenoceptor agonist, has previously been shown to reduce renal pelvic pressure during ureterorenoscopy in pigs. Now, researchers in Denmark have shown that isoproterenol reduces pelvic pressure in humans undergoing retrograde intrarenal stone surgery.

Jung et al. recruited 12 patients who were admitted for ureteroscopic kidney stone removal. Patients were randomly assigned to irrigation with saline or $0.1 \mu \mathrm{g} / \mathrm{ml}$ isoproterenol. Baseline pelvic pressure was similar (10$12 \mathrm{mmHg}$ ) in both groups, and rose to similar levels $(\sim 35 \mathrm{mmHg})$ in phase 1 of the procedure, when both groups were irrigated with saline for $5 \mathrm{~min}$. In phase 2, however, when one group received saline and the other isoproterenol, mean pelvic pressure was significantly lower in the isoproterenol group $(19 \pm 3 \mathrm{mmHg})$ than in the saline group $(33 \pm 12 \mathrm{mmHg})$. The mean 